GANIT J. Bangladesh Math. Soc. (ISSN 1606-3694) 38 (2018) 47-55

DOI: https://doi.org/10.3329/ganit.v38i0.39784

\title{
SEQUENTIALLY UPDATED WEIGHTED COST OPPORTUNITY BASED ALGORITHM IN TRANSPORTATION PROBLEM
}

\author{
A. R. M. Jalal Uddin Jamali* and Pushpa Akhtar \\ Department of Mathematics, Khulna University of Engineering \& Technology \\ Khulna 9203, Bangladesh \\ Email:armjamali@yahoo.com
}

Received: 22-03-2018 Accepted: 05-09-2018

\begin{abstract}
Transportation models are of multidisciplinary fields of interest. In classical transportation approaches, the flow of allocation is controlled by the cost entries and/or manipulation of cost entries - so called Distribution Indicator (DI) or Total Opportunity Cost (TOC). But these DI or TOC tables are formulated by the manipulation of cost entries only. None of them considers demand and/or supply entry to formulate the DI/ TOC table. Recently authors have developed weighted opportunity cost (WOC) matrix where this weighted opportunity cost matrix is formulated by the manipulation of supply and demand entries along with cost entries as well. In this WOC matrix, the supply and demand entries act as weight factors. Moreover by incorporating this WOC matrix in Least Cost Matrix, authors have developed a new approach to find out Initial Basic Feasible Solution of Transportation Problems. But in that approach, WOC matrix was invariant in every step of allocation procedures. That is, after the first time formulation of the weighted opportunity cost matrix, the WOC matrix was invariant throughout all allocation procedures. On the other hand in VAM method, the flow of allocation is controlled by the DI table and this table is updated after each allocation step. Motivated by this idea, we have reformed the WOC matrix as Sequentially Updated Weighted Opportunity Cost (SUWOC) matrix. The significance difference of these two matrices is that, WOC matrix is invariant through all over the allocation procedures whereas SUWOC matrix is updated in each step of allocation procedures. Note that here update (/invariant) means changed (/unchanged) the weighted opportunity cost of the cells. Finally by incorporating this SUWOC matrix in Least Cost Matrix, we have developed a new approach to find out Initial Basic Feasible Solution of Transportation Problems. Some experiments have been carried out to justify the validity and the effectiveness of the proposed SUWOC-LCM approach. Experimental results reveal that the SUWOC-LCM approach outperforms to find out IBFS. Moreover sometime this approach is able to find out optimal solution too.
\end{abstract}

\section{Introduction}

Transportation problem is a special case of Linear Programming. It arises many fields of physical applications includes industry, communication network, planning, scheduling transportation and allotment etc. In classical transportation approaches, like North-West Corner Rule, Least Cost 
Matrix (LCM) method [11] e.tc., the flow of allocation is controlled by the cost entries only; such as manipulation of cost entries - so called Distribution Indicator (DI) such as VAM method [9] or Total Opportunity Cost (TOC) such as Modified VAM method [4]. But these DI or TOC tables are formed by the manipulation of cost entries only. None of them considers demand and/or supply entry to formulate the DI/ TOC table.RecentlySharma and Bhadane (2016) proposed an alternative method to North-West Corner method rather than VAM method for solving transportation problem. But in this approach authors used the Statistical tool called Coefficient of Range for manipulation of cost entries only. On the other hand recently Jamali et al. (2017a) have developed weighted opportunity cost (WOC) matrix, which is off course a new idea, for the control of the flow of allocations. It is noted that this weighted opportunity cost matrix is formulated by the manipulation of supply and demand entries along with cost entries as well. In this WOC matrix, the supply and demand entries act as weight factors. Moreover by incorporating this WOC matrix in Least Cost Matrix, authors [5] have developed a new approach to find out Initial Basic Feasible Solution of Transportation Problems. But in the algorithm [6], the WOC matrix is invariant through all over the allocation procedures. On the other hand VAM method and other its variants approaches [1, 2, 3, 8 and 10] the DI/TOC matrix are changed after each step of allocations. By exploiting this idea into WOC matrix we have developed a new algorithm to find out the IBFS for the transportation problems. It is worthwhile to mention here that the partial result of this article also presented in a International Conference [7].

\section{Mathematical Formulation of WOC matrix}

As the proposed Sequentially Updated Weighted Opportunity Cost (SUWOC) matrix is developed after initial formulation of WOC matrix, so it is worthwhile to discuss briefly about the formulation of WOC matrix.

\subsection{Finding cell weight}

At first we will find out the maximum possible allocation of any cell $C_{i j}$ where transportation cost is $c_{i j}$ (unit cost from origin $i$ to destination $j$ ). Since the availability of the origin $i$ is $a_{i}$ (units) and the demand at destination $j$ is $b_{j}$ (units). So the maximum possible allocation at cell $C_{i j}$ is obviously $\min \left(a_{i}, b_{j}\right)$. Now since the maximum possible ability of allocation of each cell $C_{i j}$ is $\min \left(a_{i}, b_{j}\right)$, so the total possible maximum allocation of all cells be $\sum_{i=1}^{m} \sum_{j=1}^{n} \min \left(a_{i}, b_{j}\right)$. Therefore for each cell $C_{i j}$, its weight factor is:

$$
W_{i j}=\min \left(a_{i}, b_{j}\right) / \sum_{i=1}^{m} \sum_{j=1}^{n} \min \left(a_{i}, b_{j}\right)(1)
$$

So that $\sum_{i=1}^{m} \sum_{j=1}^{n} \boldsymbol{W}_{i j}=1$. But since the factor “ $1 / \sum_{i=1}^{m} \sum_{j=1}^{n} \min \left(\boldsymbol{a}_{i}, \boldsymbol{b}_{j}\right)$ " is common to

$\boldsymbol{W}_{\boldsymbol{i} \boldsymbol{j}} \forall \boldsymbol{i}, \boldsymbol{j}$, so we might ignore this factor. Therefore the weight factor at cell $C_{i j}$ be

$\boldsymbol{W}_{\boldsymbol{i} \boldsymbol{j}}=\boldsymbol{\operatorname { m i n }}\left(\boldsymbol{a}_{\boldsymbol{i}}, \boldsymbol{b}_{\boldsymbol{j}}\right)(2)$ It is noted that this reduces a significant amount of computational cost. 


\subsection{Finding appropriate weighted opportunity cost entries}

After successful formulation of cell weight, out next task is to formulation of weighted opportunity cost matrix. But we have a problem - since cell with lower cost has preference for allocation, on the other hand the cell with larger weight has preference for allocation. So we cannot simply multiply weight to cell cost to find meaning full elements of weighted cost matrix. To overcome this difficulty and for the formulation of meaningful weighted cost matrix, we should transform one of the two so that the multiplication of the two will be meaningful. This can be done by inversing the cost elements. Therefore the virtual weighted opportunity cost corresponding cell cost $c_{i j}$ be

$$
{ }^{\mathrm{w}} c_{i j}=\frac{1}{c_{i j}} \times \min \left(a_{i}, b_{j}\right)(3)
$$

where ${ }^{\mathrm{w}} c_{i j}$ and $c_{i j}$ denote weighted virtual cell cost and actual value of cost at the cell $c_{i j}$ respectively.

But one problem again takes place. What happen if cell cost is zero? So to prevail over this difficulty we need some more special attentions. We can overcome this shortcoming by considering zero costs and costs which is greater than zero but less than one. So if there exists any cell whose cost entry is zero, then we can formulate the weighted cost to the cell $\mathrm{C}_{\mathrm{pq}}$ as follows:

i. If $\mathrm{c}_{\mathrm{pq}}=0$ and $\left\{c_{i j}: 0<c_{i j}<1 \forall i, j\right\} \neq \Phi$ (i.e. null set), then $\operatorname{set}^{\mathrm{w}} c_{p q}=M \times \min \left(a_{i}, b_{j}\right)$

where $M=\max \left\{a_{i}, b_{j} \forall i, j\right\} /\left[\min \left\{c_{i j}: 0<c_{i j}<1 \forall i, j\right\}\right]$.

ii. Else if $\mathrm{c}_{\mathrm{pq}}=0$ and $\left\{c_{i j}: 0<c_{i j}<1 \forall i, j\right\}=\Phi$, set ${ }^{\mathrm{w}} c_{p q}=N \times \min \left(a_{i}, b_{j}\right)$

where $N=\max \left\{a_{i}, b_{j} \forall i, j\right\}$

\subsection{Algorithm of finding weighted opportunity cost (WOC) matrix}

Now we are able to overcome all the shortcomings to formulate the weighted cost. Therefore the algorithm of Weighted Cost Matrix (WOC) $\left[{ }^{\mathrm{w}} c_{p q}\right]$ is as follows:

(a) If $c_{p q}=0$ and $\left\{c_{i j}: 0<c_{i j}<1 \forall i, j\right\} \neq \Phi$ (i.e. null set), then $\operatorname{set}^{\mathrm{w}} c_{p q}=M \times \min \left(a_{i}, b_{j}\right)$ where $M=\max \left\{a_{i}, b_{j} \forall i, j\right\} /\left[\min \left\{c_{i j}: 0<c_{i j}<1 \forall i, j\right\}\right]$.

(b) Else if $c_{p q}=0$ and $\left\{c_{i j}: 0<c_{i j}<1 \forall i, j\right\}=\Phi$, set ${ }^{\mathrm{w}} c_{p q}=N \times \min \left(a_{i}, b_{j}\right)$ where $N=\max \left\{a_{i}, b_{j} \forall i, j\right\}$

(c) Else if $c_{p q}>0 \forall i, j$ then set ${ }^{\mathrm{w}} c_{p q}=\frac{1}{c_{p q}} \times \min \left(a_{i}, b_{j}\right)$

\section{Proposed Algorithm of SUWOC-LCM Method}

It is known that Least Cost Matrix (LCM) is very simple and effective to find out IBFS of TP. In LCM, the allocation flows are directly controlled by cost matrix - least cost prefers first over larger costs for allocation. In LCM, there is no any special Distribution Indicator (DI), rather than 
cost matrix, which forces the direction of flow of allocation. On the other hand VAM's method, which is more efficient to find out IBFS, has DI for control the flow of allocations. As we mentioned earlier that it's DI is formulated by the manipulation of only cost entries. Moreover it has one more significant feature - the DI table is updated after each allocation steps. It is noted that we have developed the WOC matrix by incorporating supply/demand entries as weighted factor. But we know after each allocation the amount of demand /supply is changed. So the weighted factor should be changed. By exploiting these emerging ideas, we have developed here a sequentially updated Weighted Opportunity Cost (SUWOC) matrix. Finally embedded this SUWOC upon LCM algorithm, here we have developed a modified algorithm named SUWOCLCM approach for finding IBFS of TP.

In the proposed SUWOC-LCM, the flow of allocation is controlled by the SUWOC matrix rather than cost matrix such that the cell with larger weight factor is preferred first for allocation. Moreover after each step of allocation procedures, SUWOC matrix may be changed and updated according to the present status of demand /supply entries. The algorithm of SUWOC-LCM is given below:

\section{Alg. SUWOC-LCM( )}

Step 1 (Input): read cost matrix $\left[c_{i j}\right]$, supply $\left[a_{i}\right]$ and demand $\left[b_{j}\right]$

Step 2 (Find Allocation Units): find possible maximum allocation units of each cell [ $\left.c_{i j}\right]$ : min $\left(a_{i}, b_{j}\right)$.

\section{Step 3 (Find Weighted Opportunity Cost of each Cell ${ }^{\mathrm{w}} C_{i j}$ ):}

(i) If $c_{i j}>0$, set ${ }^{\mathrm{w}} c_{i j}=\frac{1}{c_{i j}} \times \min \left(a_{i}, b_{j}\right)$

(ii) elseif $c_{i j}=0$ and $\left\{c_{p q}: 0<c_{p q}<1 \forall p, q\right\} \neq \Phi[$ null $]$ then set ${ }^{\mathrm{w}} c_{i j}=M \times \min \left(a_{i}, b_{j}\right)$

where $M=\max \left\{a_{p}, b_{q} \forall p, q\right\} /\left[\min \left\{c_{p q}: 0<c_{p q}<1 \forall p, q\right\}\right]$.

(iii) elseif $c_{i j}=0$ and $\left\{c_{p q}: 0<c_{p q}<1 \forall p, q\right\}=\Phi$, then set

${ }^{\mathrm{w}} c_{i j}=N \times \min \left(a_{i}, b_{j}\right)$ where $N=\max \left\{a_{i}, b_{j} \forall i, j\right\}$

Step 4 (Formulation of WOC matrix i.e. $\left[{ }^{\mathrm{w}} c_{i j}\right]$ : Do Step 2 and Step 3 for each $i$ and $j$ until the WOC matrix i.e. $\left[{ }^{\mathrm{w}} c_{i j}\right]$ is formed.

\section{Step 5 (Allocation procedure):}

Allocate amount of $\min \left(a_{i}, b_{j}\right)$ at cell $C_{i j}$ s.t

(i) ${ }^{\mathrm{w}} c_{i j}=\max \left\{{ }^{\mathrm{w}} c_{p q} ; \forall p, q\right\}$ and $\left|\max \left\{{ }^{\mathrm{w}} c_{p q} ; \forall p, q\right\}\right|=1$ (i.e. unique), else

(ii) ${ }^{\mathrm{w}} c_{i j}=\max \left\{{ }^{\mathrm{w}} c_{p q} ; \forall p, q\right\}$ though $\left|\max \left\{{ }^{\mathrm{w}} c_{p q} ; \forall p, q\right\}\right|>1$ (i.e. not unique), but their exist unique amount of maximum allocation i.e. $\min \left(a_{i}, b_{j}\right)$ among all possible allocations corresponding to each $\max \left\{{ }^{\mathrm{w}} c_{p q} ; \forall p, q\right\}$, else 
(iii) ${ }^{\mathrm{w}} c_{i j}=\max \left\{{ }^{\mathrm{w}} c_{p q} ; \forall p, q\right\}$ but $\left|\max \left\{{ }^{\mathrm{w}} c_{p q} ; \forall p, q\right\}\right|>1$ (i.e. not unique), and also exist multiple maximum then tie and select $C_{i j}$ among all ${ }^{\mathrm{w}} c_{i j}=\max \left\{{ }^{\mathrm{w}} c_{p q} ; \forall p, q\right\}$.

It is noted here that $\square\{.\} \square$ denotes the number of elements of the set.

\section{Step 6 (Updating transportation tableau and WOC):}

(i) If $a_{i}=\min \left(a_{i}, b_{j}\right)$ then set $a_{i}=0$ and cross out $\mathrm{c}_{i q}$ and ${ }^{\mathrm{w}} c_{i q} \forall q$ and then

(a) update demand $b_{j}{ }^{\prime}=\left|b_{j}-a_{i}\right|$;

(b) update $j^{\text {th }}$ column of WOC matrix $\left[{ }^{\mathrm{w}} c_{i j}\right]$ s.t.

$$
{ }^{\mathrm{w}} c_{p j}^{\prime}={ }^{\mathrm{w}} c_{p j} \times \frac{\min \left\{a_{p}, b_{j^{\prime}}\right\}}{\min \left(a_{p}, b_{j}\right)} \forall p
$$

(ii) Else set $b_{j}=0$ and cross out $c_{p j}$ and ${ }^{{ }^{\mathrm{w}}} c_{p j} \forall p$ and then

(a) update supply $a_{i}{ }^{\prime}=\left|b_{j}-a_{i}\right|$;

(b) update $i^{\text {th }}$ row of WOC matrix $\left[{ }^{\mathrm{w}} c_{i j}\right]$ s.t.

$$
{ }^{\mathrm{w}} c_{i q}{ }^{\prime}={ }^{\mathrm{w}} c_{i q} \times \min \left\{a_{i}{ }^{\prime}, b_{q}\right\} / \min \left(a_{i}, b_{q}\right) \forall q
$$

Step 7 (Termination condition): Repeated the Step 5 and Step 6 unless termination condition meets i.e. $a_{i}=0 \forall i$ or $b_{j}=0 \forall j$

[i.e. Continuing the allocation procedures until possible all allocations will be completed].

\section{Experiments and Discussions}

\subsection{Experiments on a Typical Example}

To justify the effectiveness and to examine the validity of the proposed algorithm we will first consider an Example 1 which is shown in the Table 1.

\begin{tabular}{|c|c|c|c|}
\hline & $D_{1}$ & $D_{2}$ & $D_{3}$ \\
\hline$O_{l}$ & 3 & 3 & 5 \\
\hline $\mathrm{O}_{2}$ & 6 & 5 & 4 \\
\hline $\mathrm{O}_{3}$ & 6 & 10 & 7 \\
\hline $\mathrm{D}$ & 7 & 12 & 8 \\
\hline
\end{tabular}

Table 1. Transportation Tableau of Ex. 1

Solution: Before allocation procedure we have to develop the WOC matrix of the example 1. Note that in this problem all the cost entries are greater than zero i.e. $c_{i j}>0$, so for initial formulation of WOC matrix we need only the formula: ${ }^{\mathrm{w}} c_{i j}=\frac{1}{c_{i j}} \times \min \left(a_{i}, b_{j}\right)$. The weighted opportunity cost (WOC) table corresponding to the given problem is shown in the Table 2. 
Table 2. WOC matrix for Ex. 1

\begin{tabular}{|c|c|c|c|}
\hline & $D_{1}$ & $D_{2}$ & $D_{3}$ \\
\hline$O_{1}$ & $7 / 3$ & $9 / 3$ & $8 / 5$ \\
\hline $\mathrm{O}_{2}$ & $7 / 6$ & $8 / 5$ & $8 / 4$ \\
\hline $\mathrm{O}_{3}$ & $7 / 6$ & $10 / 10$ & $8 / 7$ \\
\hline $\mathrm{D}$ & 7 & 12 & 8 \\
\hline
\end{tabular}

For hand calculation, we have now incorporated this WOC matrix into the corresponding Transportation Tableau (TT). The schematic view of the incorporated WOC matrix into TT is shown in the Table 3. In this table each weighted opportunity cost is given to the upper left corner of the corresponding each cell whereas each actual cost is given to the upper right corner of the corresponding each cell.

Table 3. The schematic view of WOC Matrix in TT of a TP

\begin{tabular}{|c|c|c|c|c|c|}
\hline \multirow{2}{*}{$O_{1}$} & $D_{1}$ & \multicolumn{2}{|l|}{$D_{2}$} & \multicolumn{2}{|c|}{$D_{3}$} \\
\hline & $7 / 3 \quad 3$ & $9 / 3$ & 3 & $8 / 5$ & 5 \\
\hline $\mathrm{O}_{2}$ & $7 / 6 \quad 6$ & $8 / 5$ & 5 & $8 / 4$ & 4 \\
\hline $\mathrm{O}_{3}$ & $7 / 6 \quad 6$ & $10 / 10 \quad 1$ & 10 & $8 / 7$ & 7 \\
\hline
\end{tabular}

Now we have allocated to each cell according to the SUWOC-LCM approach. The complete solution according to the SUWOC-LCM approach of the problem is shown in the table 4 .

Table 4: The complete solution of the TP Ex.1

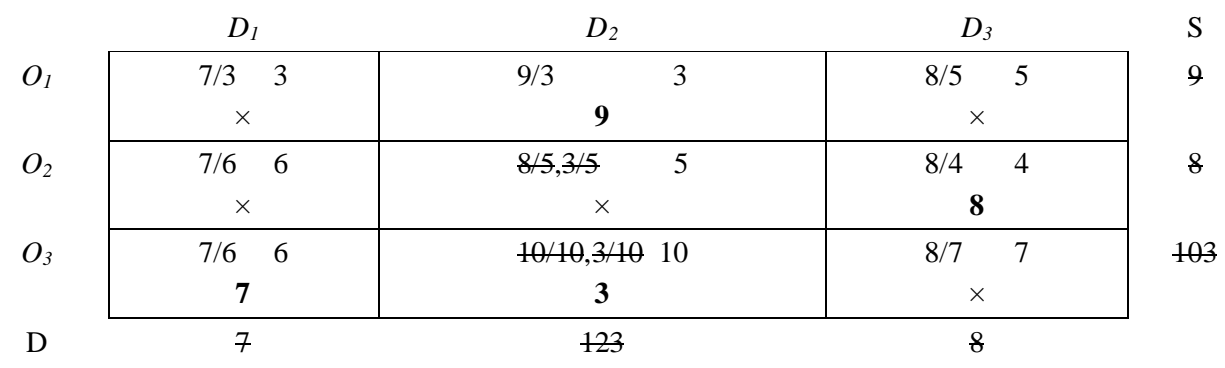

Therefore total cost (by SUWOC based LCM approach) $=9 \times 3+8 \times 4+7 \times 6+3 \times 10=\mathbf{1 3 1}$

Now to justify the effectiveness as well as efficiency of the proposed algorithm, a comparison study for this numerical example is done. For the comparison we have considered LCM and along with the proposed SUWOC-LCM approach. The comparison results are displayed in the Table 5. It is observed that the proposed SUWOC matrix based LCM approach outperforms to other approaches. It is also observed that the IBFS of the proposed method is not optimal but relatively near to optimal solution. So we will need cheaper computational cost to get optimal solution by MOD or other optimal procedures by using IBFS obtained by the proposed method. 
Table 5. The comparison among LCM, WOC-LCM, VAM, and SUWOC based LCM approach

\begin{tabular}{|l|c|c|c|c|}
\hline Method & LCM & VAM & SUWOC-LCM & Optimal Value \\
\hline Total Cost & 159 & 143 & $\mathbf{1 3 1}$ & $\mathbf{1 2 5}$ \\
\hline
\end{tabular}

\subsection{Further experiments and discussions}

Now to compare the performance of the proposed SUWOC-LCM method to existing approaches we have performed further experiments. For this experimental study we have considered some instances given in the first column of the Table 6. For the comparison study we consider two wellknown approach namely LCM and VAM methods. The experimental results are display in the Table 6. In the Table 6, SUWOC-LCM denotes the proposed Sequentially Updated Weighted Opportunity Cost Matrix based Least Cost Method, LCM means Least Cost Matrix method and VAM indicates Vogel's Approximation Method.

Table 6: Comparison of SUWOC_LCM, LCM and VAM approaches in TP

\begin{tabular}{|c|c|c|c|c|c|}
\hline $\begin{array}{l}\text { Ex. } \\
\text { No. }\end{array}$ & Problem & $\begin{array}{l}\text { SUWOC- } \\
\text { LCM }\end{array}$ & LCM & VAM & $\begin{array}{c}\text { Optimal } \\
\text { Value }\end{array}$ \\
\hline 1 & $\begin{array}{l}c_{i j}:\{(5,7,9,11) ;(7,9,11,13) ;(9,11,13,15) ;(11,13,15,17)\} \\
S:(10,25,30,35) \\
D:(20,30,15,35)\end{array}$ & 1210 & 1210 & 1210 & 1210 \\
\hline 2 & $\begin{array}{l}c_{i j}:\{(6,4,1) ;(3,8,7) ;(4,4,2)\} \\
S:(50,40,60) \\
D:(20,95,35)\end{array}$ & 555 & 555 & 555 & 555 \\
\hline 3 & $\begin{array}{l}c_{i j}:\{(2,4,1,3) ;(4,3,5,2) ;(5,2,3,6)\} \\
S:(10,20,10) \\
D:(9,11,6,14)\end{array}$ & 85 & 85 & 85 & 85 \\
\hline 4 & $\begin{array}{l}c_{i j}:\{(1,2,3,4,5) ;(2,3,4,5,6) ;(3,4,5,6,7) ;(4,5,6,7,8)(5,6,7,8,9)\} \\
S:(10,25,15,20,30) \\
D:(20,10,5,30,35)\end{array}$ & 585 & 585 & 585 & 585 \\
\hline 5 & $\begin{array}{l}c_{i j}:\{(9,8,5,7) ;(4,6,8,7) ;(5,8,9,5)\} \\
S:(12,14,16) \\
D:(8,18,13,3)\end{array}$ & 240 & 248 & 248 & 240 \\
\hline 6 & $\begin{array}{l}c_{i j}:\{(4,19,22,11) ;(1,9,14,14) ;(6,6,16,14)\} \\
S:(100,30,70) \\
D:(40,20,60,80)\end{array}$ & 2160 & 2090 & 2170 & 2040 \\
\hline 7 & $\begin{array}{l}c_{i j:}:\{(9,12,9,6,9,10) ;(7,3,7,7,5,5) ;(6,5,9,11,3,11) ;(6,8,11,2,2,10)\} \\
S:(5,6,2,9) \\
D:(4,4,6,2,4,2)\end{array}$ & 112 & 114 & 112 & 112 \\
\hline 8 & $\begin{array}{l}c_{i j}:\{(4,5,8,4) ;(6,2,8,1) ;(8,7,9,10)\} \\
S:(52,57,54) \\
D:(60,45,8,50)\end{array}$ & 795 & 674 & 674 & 674 \\
\hline
\end{tabular}




\begin{tabular}{|c|l|c|c|c|c|}
\hline 9 & $c_{i j}:\{(5,2,4,1) ;(5,2,1,4) ;(6,4,8,2) ;(4,6,5,4)(2,8,4,5)\}$ & 429 & 423 & 391 & 381 \\
& $\begin{array}{l}S:(30,20,12,30,46) \\
D:(31,50,30,27)\end{array}$ & & & \\
\hline 10 & $c_{i j}:\{(2,5,4) ;(6,1,2) ;(4,5,2)\}$ & 29 & 29 & 29 & 29 \\
& $\begin{array}{l}\text { S: }(4,6,6) \\
D:(3,7,6)\end{array}$ & & & & \\
\hline 11 & $\begin{array}{l}c_{i j}:\{(19,30,50,10) ;(70,30,40,60) ;(40,8,70,20)\} \\
S:(7,9,18) \\
D:(5,8,7,14)\end{array}$ & 779 & 814 & 779 & 743 \\
\hline 12 & $\begin{array}{l}c_{i j}:\{(10,0,20,11) ;(12,7,9,20) ;(0,14,16,18)\} \\
S:(20,25,15) \\
D:(10,15,15,20)\end{array}$ & 480 & 480 & 480 & 460 \\
\hline 13 & $\begin{array}{c}c_{i j}:\{(3,3,5) ;(6,5,4) ;(6,10,7)\} \\
S:(90,80,100) \\
D:(70,120,80)\end{array}$ & 1310 & 1590 & 1430 & 1250 \\
\hline
\end{tabular}

It is observed in the Table 6 that out of 13 instances, the proposed SUWOC-LCM outperform in 2 instances namely Ex. No. 5 and 13 compared to both LCM and VAM methods. It is also noticed that in two cases (Ex. No. 7 and 11) the results of VAM and proposed method are identical but better that of LCM method. Again in one case namely Ex. No. 5, the solutions of SUWOC-LCM is optimal and the value is better than that of other two approaches. In Ex. 6 the proposed method performed better than VAM method but worse than LCM method. In six cases namely Ex. 1, 2, 3, 4, 10 and 12, the results of all the three methods are identical. But it is also notice that in Ex. No. 8 and Ex. No. 9 the results of the proposed method are worst compared to other two methods.

From these primary experimental investigations, we may conclude that the performance of the proposed algorithm comparatively better than both LCM and VAM method. But it is noted that solution obtained by the proposed method is IBFS and there is no any guaranty that the solution to be optimal.

\section{Conclusion}

In this study, we have developed a Sequentially Updated Weighted Opportunity Cost (SUWOC) matrix on the basis of WOC matrix. At first we have formulated initial WOC matrix then the initial WOC matrix is sequentially updated after each step of allocations during the allocation procedures and it is named as SUWOC matrix. It is noted that the initial WOC as well as SUWOC are formulated by the manipulation of supply and demand entries along with cost entries where supply and demand entries are treated as weight factors. But SUWOC matrix is updated in each step of allocations. On the basis of the experimental study, we may conclude that sequentially updated weighted Opportunity Cost (SUWOC) matrix based LCM approach is much more efficient than LCM approach and comparable with VAM approach. Moreover the idea of WOC/SUWOC, which is formulated by considering demand/supply, is unique and we may say that this is a new window. The researchers can view through this new window for solving the TP problems. 


\section{REFERENCES}

[1] Azad S. M. A. K., Hossain M. B. and Rahman M. M., An algorithmic approach to solve transportation problems with the average total opportunity cost method, International Journal of Scientific and Research Publications, ISSN: 2250-3153, 7(2), 266-269, 2017.

[2] Deshmukh N. M., An innovative method for solving transportation problem, International Journal of Physics and Mathematical Sciences ISSN: 2277-2111 (Online), 2(3), July-September, 86-91, 2012.

[3] Hakim M. A., An alternative method to find initial basic feasible solution of transportation problem, Annals of Pure and Applied Mathematics, 1(2), 203-209, 2012.

[4] Islam M. A., Cost and time minimization in transportation and maximization of profit: A linear programming approach, Ph.D. Thesis, Department of Mathematics, Jahangirnagar University, 2012.

[5] Jamali, A. R. M. Jalal Uddin, Pushpa A. and Fatima J., Weighted Cost Based Distribution Opportunity Table in Transportation Problems, Proceedings of the International Conference on Mechanical Engineering and Renewable Energy 2017, (ICMERE 2017), 18 - 20 December, 2017a, CUET, Chittagong, Bangladesh.

[6] Jamali A. R. M. Jalal Uddin, Pushpa A. and Fatima J.,Weighted cost opportunity based algorithm for initial basic feasible solution: A new approach in transportation problem, Journal of Engineering Science, 8(1), 63-70, 2017b.

[7] JamaliA. R. M. Jalal Uddinand Pushpa A., Sequentially Updated Weighted Opportunity Cost Matrix for Allocation Flow in Transportation Problems, $20^{\text {th }}$ International Mathematics conference 2017, 0810 December, 2017, DU, Dhaka.

[8] Korukoglu S. and Balli S., An improved Vogel's approximation method for the transportation problem, Association for Scientific Research,Mathematical and Computational Application, 16(2), 370-381,2011.

[9] Reinfeld N .V, and W. R. Vogel, Mathematical Programming, Englewood Gliffs, New Jersey: Prentice-Hall, 1958.

[10] Sharma N. M. and Bhadane A. P., An alternative method to north-west corner method for solving transportation problem, International Journal for Research in Engineering Application \& Management (IJREAM), ISSN: 2494-9150, 1(12), 1-3, 2016.

[11] Taha A. H., Operation Research an Introduction, 7th Ed., India, Prentice-Hall, 2003. 